\title{
Biomarcadores inflamatórios e de estresse oxidativo em cadelas submetidas à ovário- histerectomia videoassistida ou convencional
}

\author{
[Inflammation and oxidative stress indicators in bitches submitted to laparoscopic-assisted \\ or conventional ovariohysterectomy] \\ F. Dalmolin ${ }^{1}$, C.L. Lhamas ${ }^{2}$, S.T.L. Pinto Filho ${ }^{2}$, J.P.S. Feranti ${ }^{2}$, A. Poerschke, \\ R.C. Beck ${ }^{3}$, F.H. Abdalla ${ }^{2}$, C.M. Andrade ${ }^{4}$, M.V. Brun ${ }^{4 *}$ \\ ${ }^{1}$ Fundação Universidade Regional de Blumenau - FURB - Blumenau, SC \\ ${ }^{2}$ Programa de pós-graduação - Universidade Federal de Santa Maria - UFSM - Santa Maria, RS \\ ${ }^{3}$ Aluno de graduação - Universidade Federal de Santa Maria - UFSM - Santa Maria, RS \\ ${ }^{4}$ Universidade Federal de Santa Maria - UFSM - Santa Maria, RS
}

\begin{abstract}
RESUMO
A videocirurgia é atualmente uma das principais ferramentas operatórias, com vantagens que incluem menor estresse, incisões e dor pós-operatória quando comparada aos procedimentos abertos. Objetivou-se comparar o processo inflamatório e o estresse oxidativo resultantes das técnicas de ovário-histerectomia $(\mathrm{OVH})$ convencional e videoassistida, com dois portais em cadelas, por meio de hemograma, avaliação de acetilcolinesterase, butirilcolinesterase, catalase e malondialdeído séricos, imediatamente antes da operação e duas, seis, 12, 24, 48 e 72 horas após a cirurgia. Observou-se menor estresse cirúrgico nas pacientes operadas pela técnica videoassistida, e sugere-se que a técnica convencional possa implicar peroxidação lipídica, mesmo com o uso de anti-inflamatório.
\end{abstract}

Palavras-chave: espécies reativas, videocirurgia, acetilcolinesterase, butirilcolinesterase, peroxidação lipídica

\begin{abstract}
Videosurgery is currently a very important surgical tool with several benefits over the open surgery, including less surgical stress, shorter incisions and less postoperative pain. The purpose of this study was to compare the inflammatory process and oxidative stress between conventional and two-port laparoscopic-assisted ovariohisterectomy $(\mathrm{OVH})$ in bitches. Complete blood counting, serum acetylcholinesterase, butyrylcholinesterase, catalase and malondialdehyde were assessed on the baseline and at two, six, 12, 24, 48 and 72 hours after surgery. The patients submitted to the videoassisted technique presented lower inflammatory response. There are suggestions that the conventional technique promotes lipid peroxidation, even with the use of anti-inflammatories.
\end{abstract}

Keywords: reactive species, videosurgery, acetylcholinesterase, butyrylcholinesterase, lipid peroxidation

\section{INTRODUÇÃO}

Ao longo dos últimos 20 anos, a laparoscopia tornou-se importante ferramenta operatória para pequenos animais. Inicialmente utilizada para fins diagnósticos, atualmente, na medicina, permite realizar a grande maioria das cirurgias previamente executadas por laparotomia (Guven,

Recebido em 13 de março de 2015

Aceito em 15 de novembro de 2015

* Autor para correspondência (corresponding author)

E-mail: fabiola.dalmolin@uffs.edu.br
2010). Abordagens laparoscópicas resultam em menor estresse que procedimentos abertos (Freeman et al., 2010), além de diminuir o tempo de internação hospitalar e de dor pós-operatória, bem como permitir rápido retorno às atividades cotidianas (Lee e Kim, 2014).

O estresse apresenta infinidade de formas e é integrante de todas as doenças e traumas. A resposta ao estresse envolve hormônios, 
citocinas, alterações na função celular e efeitos sobre a neurotransmissão (Thomas e Balasobramanian, 2004). Entre os fatores envolvidos, são citadas as catecolaminas, as citocinas, as proteases, as espécies reativas, os eicosanoides, as proteínas de fase aguda, os fatores de crescimento e os hormônios adrenocorticotróficos (Kim e Deutschman, 2000). O hemograma de estresse é resposta orgânica que pode ser mediada pela liberação de cortisol em doenças sistêmicas, distúrbios metabólicos e dor (Trall, 2007) e caracteriza-se por resposta leucocitária com neutrofilia, linfopenia, monocitose e eosinopenia (Meyer et al., 1995).

As enzimas acetilcolinesterase (AChE) e butirilcolinesteras (BChE) séricas, por sua vez, foram utilizadas como indicadores da inflamação de baixo grau (Das, 2007). Essas enzimas foram mensuradas em cães submetidos a diferentes técnicas de $\mathrm{OVH}$ e se mostraram capazes de detectar esse tipo de inflamação (Basso et al., 2014).

Espécies reativas são átomos, moléculas ou íons altamente instáveis e ativos para reações químicas. $\mathrm{O}$ equilíbrio entre a produção de espécies reativas e a neutralização por antioxidantes é delicado; quando o desequilíbrio tende à superprodução, as células sofrem estresse oxidativo, participante da patogênese de doenças cardiovasculares, neuropáticas, renais, hepáticas, inflamatórias e de neoplasmas (Carocho e Ferreira, 2013).

Considerando que o trauma cirúrgico pode promover alterações orgânicas (Arsalani-Zadeh et al., 2011), objetivou-se comparar a resposta inflamatória e o estresse oxidativo resultantes das técnicas de $\mathrm{OVH}$ convencional e videoassistida por dois portais, em cadelas medicadas com antiinflamatórios.

\section{MATERIAL E MÉTODOS}

Este trabalho foi aprovado pela Comissão de Ética no Uso de Animais em Ensino e Pesquisa (CEUA), sob parecer 081/2012(4).

Foram selecionadas fêmeas caninas por meio de anamnese, exames clínico, laboratoriais e ultrassonográfico abdominal. $\mathrm{O}$ último foi indicado nos casos suspeitos de alterações reprodutivas ou gestação. Os exames laboratoriais foram analisados de acordo com os valores de referência de Rizzi et al. (2010). No hemograma, foram considerados o eritrograma (hematócrito: 37-55\%; contagem total de eritrócitos: $5,5-8,5 \times 10^{6} / \mu \mathrm{L}$ e hemoglobina: 12 $18 \mathrm{~g} / \mathrm{dL}$ ), o leucograma (contagem total de leucócitos: $6000-17000 / \mu \mathrm{L})$ e a contagem de plaquetas: $200.000-5000.000 / \mu \mathrm{L}$. No diferencial de leucócitos, foram considerados os neutrófilos segmentados $(3.000-11.500 / \mu \mathrm{L})$, os linfócitos $(1.000-4.800 / \mu \mathrm{L})$, os monócitos $(150-1350 / \mu \mathrm{L})$, os eosinófilos $(100-1250 / \mu \mathrm{L})$ e os basófilos (raros). Com relação aos exames bioquímicos, estes foram analisados conforme os valores propostos por Kaneko (2008), sendo avaliadas proteínas plasmáticas totais $(5,4-7,1 \mathrm{mg} / \mathrm{dL})$, albumina $\quad(2,6-3,3 \mathrm{~g} / \mathrm{dL}), \quad$ creatinina $(0,5-$ $1,5 \mathrm{mg} / \mathrm{dL})$, fosfatase alcalina sérica (20-156 UI/L) e alanina aminotransferase (21-102UI/L).

Assim, foram incluídas no estudo 14 cadelas hígidas, adultas até quatro anos de idade, com massa corpórea média de $15,83 \pm 3,77 \mathrm{~kg}$, livres de alterações clínicas, laboratoriais e sem condições patológicas associadas ao sistema reprodutivo. A fím de confirmar a última condição, após a cirurgia, os úteros foram avaliados por meio de exames macro e microscópicos (histopatológico).

As pacientes foram internadas 48 horas antes da cirurgia no local, onde se realizaram posteriormente as avaliações pós-operatórias, para ambientação. $\mathrm{Na}$ ocasião, receberam $1 \mathrm{mg} . \mathrm{kg}^{-1}$ de nitenpiram (via oral) (Capstar ${ }^{\circledR}$, Novartis, Brasil), além de água e ração comercial ad libitum; foram ainda colocadas em contato com o ambiente climatizado, as pessoas envolvidas, os outros animais e com o procedimento de avaliação. Após jejum préoperatório sólido de 12 horas e hídrico de oito horas, realizou-se divisão aleatória em dois grupos por sorteio da técnica cirúrgica. Os animais foram tranquilizados com acepromazina $\left(0,05 \mathrm{mg} \cdot \mathrm{kg}^{-1}\right)$ (Acepran $1 \% \AA$, Vetnil, Brasil) e induzidos com propofol (4 mg. $\mathrm{kg}^{-1}$ ) (Diprivan $1 \% \AA$, Cristália, Brasil). Logo após, foram intubados e mantidos com isoflurano (Isoforine $\AA$, Cristália, Brasil) em oxigênio $100 \%$, sendo mantidos no estágio III, plano três de Guedel (Massone, 2011). Foi associada infusão intravenosa contínua de citrato de fentanila $\left(15 \mu \mathrm{g} \cdot \mathrm{kg}-1 . \mathrm{h}^{-1}\right) \quad$ (Fentanest, Cristália, Brasil), regulada por bomba de infusão 
peristáltica rotativa, 15 minutos antes do início do procedimento sem uso de administração em bolus. A aplicação de ampicilina sódica (20mg.kg ${ }^{-1}$, i.v.) (Amplatil 1g, Nova Farma, Brasil) foi realizada 30 minutos antes da cirurgia, e a fluidoterapia instituída com solução de ringer lactato de sódio $\left(10 \mathrm{ml} . \mathrm{kg}^{-1} \cdot \mathrm{h}^{-1}\right.$, i.v.) (Ringer com lactato, Sanobiol, Brasil) até a extubação.

Todos os animais foram operados pelo mesmo cirurgião, proficiente em ambas as técnicas, utilizando o mesmo padrão cirúrgico. No grupo GC (grupo convencional), realizou-se OVH por celiotomia mediana, iniciando na cicatriz umbilical e estendendo-se um terço da distância entre a cicatriz e o púbis (terço cranial do abdômen caudal). Foram realizadas ligaduras duplas (circular e transfixante) nos pedículos ovarianos e no coto uterino, com poliglactina 2-0 (Poliglatina 910, Bioline, Brasil), conforme a técnica das três pinças, modificada com ruptura manual do ligamento suspensório (Macphail, 2013). No GV, o acesso ao abdômen foi realizado com dois portais de $11 \mathrm{~mm}$, sendo um inserido na região da cicatriz umbilical e outro na região pré-púbica (Ataíde et al., 2010); utilizaram-se pneumoperitônio com pressão de $12 \mathrm{mmHg}$ e cauterização bipolar para os pedículos ovarianos e ligaduras duplas no coto com o referido fio. A dermorrafia foi realizada com náilon 3-0 (Nylon 3-0, Technofio, Brasil) e pontos em Wolf. Ao término da cirurgia, as pacientes de ambos os grupos foram medicadas (i.v.) com a associação dipirona (25mg.kg-1) e escopolamina $\left(0,2 \mathrm{mg} \cdot \mathrm{kg}^{-1}\right) \quad$ (medicamento genérico, Farmace, Brasil) e meloxicam (0,2mg. $\left.\mathrm{kg}^{-1}\right)$ (Maxicam 0,2\%, Ourofino, Brasil); as doses subsequentes foram aplicadas q.i.d. (s.c.) e s.i.d (s.c.), respectivamente, completando 48 horas de tratamento. Os úteros e os ovários foram fixados em formol $10 \%$ e enviados para avaliação histopatológica.

Foram coletadas amostras de sangue imediatamente antes das cirurgias, por punção direta, e às duas, seis, 12, 24, 48 e 72 horas de pós-operatório, por meio de cateter implantado na veia jugular com o paciente sob anestesia. Para o leucograma, utilizaram-se amostras coletadas com EDTA, processadas conforme Jain (1993), sendo considerados os valores de referência propostos por Rizzi et al. (2010). Para as demais mensurações, foram considerados os valores basais de cada paciente. A atividade da
BChE foi determinada com soro obtido pela centrifugação de sangue acondicionado em tubos sem anticoagulante, conforme Ellman et al. (1961). Para AChE, utilizou-se sangue total com EDTA e ensaio pelo método de Ellman et al. (1961), modificado por Worek et al. (1999). A atividade da catalase (CAT) foi obtida utilizando-se sangue total e citrato de sódio, pelo método modificado de Nelson e Kiesow (1972). A peroxidação lipídica foi determinada por meio de soro (conforme descrição anterior), pela técnica de TBARS (substâncias reativas com o ácido tiobarbitúrico), conforme Jentzsch et al. (1996). As proteínas dos ensaios enzimáticos foram mensuradas segundo Bradford (1976), utilizando-se albumina bovina como padrão. Exceto as amostras do leucograma, todas foram congeladas a $-20^{\circ} \mathrm{C}$ e, após, mantidas a $-80^{\circ} \mathrm{C}$ até a análise.

Todos os dados foram submetidos ao teste de normalidade de Shapiro-Wilk. Posteriormente, foram comparados entre os diferentes momentos pelo teste $\mathrm{F}$ da Análise de Variância (ANOVA). A fim de se compararem as variáveis, utilizou-se o teste não paramétrico de Kruskal-Wallis e, para as médias dos grupos, o teste Scott-Knott. As análises foram realizadas por programação no software R Core... (2014), sendo considerado nível de significância de 5\%.

\section{RESULTADOS E DISCUSSÃO}

Objetivando-se não haver interferência de patologias que pudessem alterar os resultados da avaliação inflamatória e oxidativa das técnicas cirúrgicas, foram selecionadas cadelas hígidas por meio de anamnese, exames clínico, laboratoriais e ultrassonográfico, sendo o último realizado caso houvesse suspeita de alterações reprodutivas ou gestação. Adicionalmente, os úteros foram avaliados macro e microscopicamente, confirmando não existirem alterações nestes, que poderiam interferir na avaliação da resposta inflamatória bem como no estresse oxidativo. Dessa forma, foram incluídas no estudo 14 cadelas hígidas.

O estresse promove reações orgânicas, ocasionando modificações dos mecanismos fisiológicos, que objetivam manter a homeostase. Apesar de essas reações serem necessárias para a proteção da homeostasia, elas contêm elementos 
que podem aumentar a susceptibilidade às doenças (Breazile, 1998).

Verificou-se leucocitose ao hemograma, com magnitude semelhante, em ambos os grupos (Fig. 1). São resultantes da anestesia e de trauma cirúrgico alterações desfavoráveis na homeostase corporal, as quais podem ser coletivamente referidas como estresse cirúrgico (Lee e Kim, 2014), que cursa como resposta inflamatória local, sistêmica, endócrina, metabólica, humoral e como ativação imunológica de vários sistemas (Kücükakin, 2009). A inflamação, um dos mais importantes e úteis mecanismos de defesa, envolve constituintes celulares, humorais e teciduais após lesão celular ou tecidual. Leucócitos, neutrófilos, eosinófilos, basófilos, mastócitos, monócitos, macrófagos, linfócitos e células do plasma contribuem para migração, liberação de enzimas e atuam como mediadores inflamatórios, além de induzirem fagocitose (Vajdovich, 2008).

Observou-se, após a liberação de cortisol, neutrofilia devido à liberação de neutrófilos da medula óssea, menor migração tecidual e diminuição da aderência à parede vascular (Stockham e Scott, 2002). A liberação de glicocorticoides endógenos pode induzir leucocitose no cão e a contagem de leucócitos nessa situação normalmente varia de 15.000$25.000 / \mu \mathrm{L}$ de sangue (Schultze, 2010), conforme observado no presente estudo, nos tempos seis e 12 horas, no GC e no GV.

Realizou-se a contagem diferencial de leucócitos, e, na contagem dos neutrófilos, houve, tanto no GC como no GV, aumento da contagem em seis e 12 horas e redução às 24 horas, sendo verificados valores estatisticamente semelhantes aos basais às 48 e às 72 horas. Entre os grupos, houve diferença em seis horas $(\mathrm{P}=0,01)$, com menor contagem celular no GV (Fig. 1). Quando há resposta sistêmica, observa-se, muitas vezes, aumento nos leucócitos circulantes no sangue (Vajdovich, 2008), conforme verificado em ambos os grupos em seis, 12 e 24 horas, mesmo com o uso de anti-inflamatório. A menor intensidade de resposta no $\mathrm{GV}$ pode ser justificada, conforme sugestão de Desborough (2000), pelo fato de que a videocirurgia provoca menor injúria tecidual que os procedimentos abertos.
Observou-se no GC diminuição significativa na contagem de linfócitos às duas e às seis horas de pós-operatório, com retorno aos valores basais às 12 horas. No GV, essas variações não foram observadas entre os tempos. Entre os grupos, houve diferença em seis horas $(\mathrm{P}=0,01)$, com menor contagem no GC (Fig. 1). Em cães, a contagem de linfócitos entre os valores de 700$1500 / \mu \mathrm{L}$ é consistente com os efeitos de glicocorticoides (Rebar, 2004), conforme verificado no GC deste estudo, corroborando os resultados observados na contagem de neutrófilos de que a videocirurgia é menos traumática que a cirurgia aberta (Desborough, 2000).

Com relação aos eosinófilos, não foram verificadas alterações no $\mathrm{GV}$; no $\mathrm{GC}$, houve diferença entre os tempos, com diminuição da contagem em duas, seis e 12 horas, e retorno aos valores normais 24 horas após o procedimento. Foi observada diferença entre os grupos em duas, seis, 12 e 24 horas $(\mathrm{P}=0,01 ; \mathrm{P}<0,00, \mathrm{P}<0,00 \mathrm{E}$ $\mathrm{P}<0,00$, respectivamente), sendo as menores contagens observadas no GC (Fig. 1).

A resposta leucocitária com neutrofilia madura leve, linfopenia, eosinopenia e monocitose moderada caracteriza o hemograma de estresse (Rebar, 2004). Essa resposta orgânica é mediada pela liberação de cortisol (Trall, 2007). À exceção da monocitose, a neutrofilia madura leve, a linfopenia e a eosinopenia foram observadas no GC deste estudo, indicando maior estresse nos animais submetidos à técnica convencional de $\mathrm{OVH}$ quando comparados àqueles submetidos à videoassistida.

A neutrofilia madura é resultado de vários fatores, que incluem a diminuição da migração de neutrófilos do sangue para os tecidos, o aumento da liberação de neutrófilos maduros mediante pool de armazenamento da medula óssea, assim como a diminuição da marginalização de neutrófilos. Já a linfopenia é atribuída à redistribuição de linfócitos circulantes, e a monocitose é resultado da mobilização de células dentro dos vasos sanguíneos. A eosinopenia ocorre devido à inibição da liberação de eosinófilos da medula óssea e ao sequestro de eosinófilos nos tecidos (Schultze, 2010). 
Biomarcadores inflamatórios...
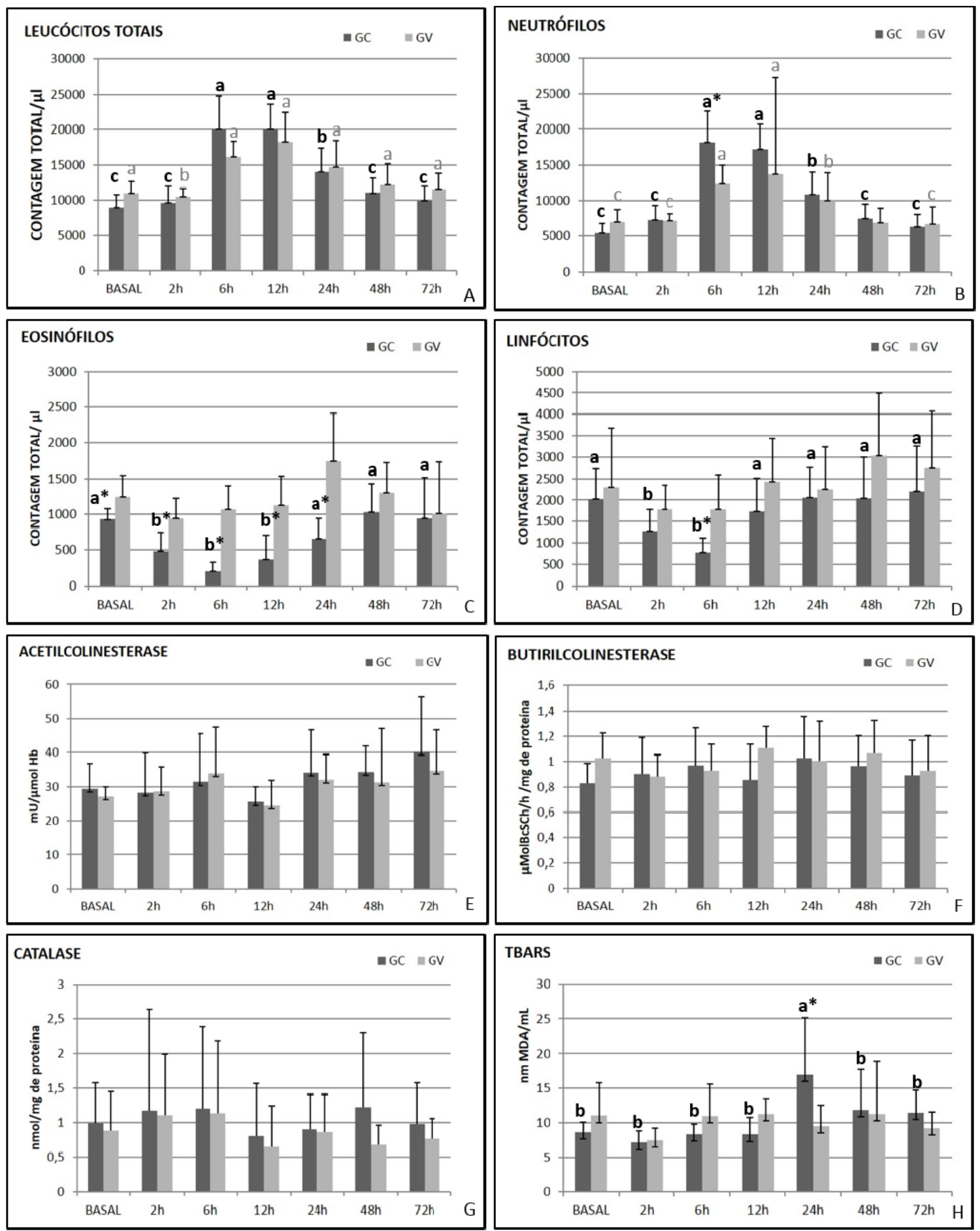

Figura 1. Médias e desvios-padrão dos parâmetros inflamatórios e de estresse oxidativo de cadelas submetidas à $\mathrm{OVH}$ videoassistida com dois portais ou convencional. Contagem total de leucócitos (A), neutrófilos (B), eosinófilos (C) e linfócitos (D). Atividade sérica da acetilcolinesterase (E), butirilcolinesterase $(\mathrm{F})$, catalase $(\mathrm{G})$ e malondialdeído (TBARS) $(\mathrm{H})$. Letras diferentes na mesma figura demonstram diferenças significativas entre os grupos avaliados $(\mathrm{P}<0,05)$. 
Considerando que a acetilcolina tem papel na supressão da inflamação, a mensuração das colinesterases, que regulam a concentração dessa enzima, pode ser útil a fim de identificar a presença de inflamação, mesmo em baixo grau (Ulloa, 2005). Basso et al. (2014) identificaram aumento dos valores basais da AChE e BChE após OVH pela técnica aberta em caninos, sem uso de anti-inflamatórios. No presente estudo, foi utilizado meloxicam e não se verificou aumento na atividade dessas enzimas em ambos os grupos (GC e GV), diferentemente do observado por estes autores anteriormente citados. Acredita-se que o fármaco tenha modulado a produção de citocinas pró-inflamatórias, não sendo verificadas alterações significativas das referidas enzimas no atual estudo.

Lesões peritoneais causam liberação de plaquetas, fibrinogênio, histamina, e cininas vasoativas. A geração de espécies reativas também é observada, e a remoção inadequada e/ou os altos níveis desses produtos resultam do estresse oxidativo, que pode alterar o funcionamento metabólico, causar dano celular (Souza et al., 2003) e provocar mudanças permanentes no material genético. Ainda, tem-se conhecimento da correlação dessas moléculas com processos patológicos (Vajdovich, 2008), envelhecimento (Vasconcelos et al., 2007), mutagênese e carcinogênese (Vajdovich, 2008).

Não foram verificadas diferenças significativas entre os tempos nem entre os grupos com relação às mensurações da CAT (Fig. 1), fato que pode ser associado ao uso de anti-inflamatório. A constatação de níveis plasmáticos dessa enzima inferiores aos do pré-operatório poderia sugerir que o processo inflamatório causado pelo trauma cirúrgico tenha sido de tamanha intensidade que poderia ter ocasionado a exaustão dessa enzima (Murugan e Pari, 2007), condição não observada no presente trabalho. Entretanto, deve-se considerar que a CAT, a principal enzima responsável pela detoxificação celular e oxidação de ácidos graxos de cadeia longa, é apenas uma das enzimas da defesa antioxidante. Dessa maneira, não se pode afirmar que não houve estresse oxidativo baseando-se apenas em um resultado isolado, já que este poderia ser investigado mediante mensuração de enzimas, como a superóxido dismutase e a glutationa peroxidase (Vasconcelos et al., 2007). Assim, sugere-se associar essas variáveis em outros estudos para melhor elucidação dos resultados.

Não se observaram diferenças das mensurações do MDA no GV. No GC, entretanto, verificou-se diferença entre os tempos e entre os grupos às 24 horas $(\mathrm{P}=0,04)$ (Fig. 1). $\mathrm{O}$ MDA, produto secundário da peroxidação lipídica, é derivado da ruptura de ácidos graxos poli-insaturados (ácido linoleico, araquidônico e docosaexanoico), sendo considerado potencial biomarcador geral de dano oxidativo lipídico no plasma (Vasconcelos et al., 2007). O aumento pontual da mensuração do MDA pode sugerir peroxidação lipídica no GC, fato não observado nos animais operados pela técnica videoassistida. No entanto, esse parâmetro deve ser mais bem investigado, por meio de aferições adicionais de outros biomarcadores em estudos subsequentes.

Embora tenha sido necessário maior tempo para a realização da técnica videoassistida $(\mathrm{P}=0,01$; $\mathrm{GV}=31,87 \pm 7,41 \mathrm{~min}$ e $\mathrm{GC}=22,12 \pm 3,52 \mathrm{~min}$ ), não foi observado estresse oxidativo nesse grupo. Como se tem conhecimento, a duração da cirurgia e o tempo de isquemia/reperfusão determinam a formação de espécies reativas e o grau de estresse oxidativo (Kücükakin, 2009; Guven, 2010). Assim, acredita-se que o tempo cirúrgico não tenha sido tão prolongado para o procedimento executado, a ponto de causar essa alteração, e ainda, que o balanço oxidativo possa ter sido atenuado pela utilização de antiinflamatórios.

\section{CONCLUSÃO}

A técnica de $\mathrm{OVH}$ videoassistida por dois portais em caninos causa menor estresse cirúrgico que a técnica convencional, e sugere-se que a última possa implicar peroxidação lipídica.

\section{AGRADECIMENTO}

À empresa WEM Equipamentos Eletrônicos Ltda., pelo fornecimento das pinças bipolares (Lina Tripol Power Blade), utilizadas nos procedimentos de $\mathrm{OVH}$ videoassistidas. 


\section{REFERÊNCIAS}

ARSALANI-ZADEH, R. ULLAH, S.; KHAN, S.; MACFIE, J. Oxidative stress in laparoscopic versus open abdominal surgery: a systematic review. J. Surg. Res., v.169, p.59-68, 2011.

ATAIDE, M.W., BRUN, M.V.; BARCELOS, L.J.G. et al. Ovariosalpingohisterectomia vídeoassistida ou convencional em cadelas com o uso de Ligasure atlas ${ }^{\mathrm{TM}}$. Cienc. Rural, v.40, p.19741979, 2010.

BASSO, P.C. RAISER, A.G.; BRUN, M.V. et al. Biomarcadores inflamatórios e indicadores de estresse oxidativo em cadelas submetidas à ovariossalpingohisterectomia convencional, por NOTES híbrida e NOTES total. Cienc. Rural, v.44, p.884-890, 2014.

BRADFORD, M.M. A rapide and sensitive method for quantification of microgram quantities of protein utilizing the principle of protein-dye binding. Anal. Biochem., v.72, p.248-254, 1976.

BREAZILE, J.E. The physiology of stress and its relationship to mechanisms of disease and therapeutics. Vet. Clin. N. Am: Food Anim. Pract., v.4, p.441-478, 1988.

CAROCHO, M.; FERREIRA, I.C.F.R. A review on antioxidants, prooxidants and related controversy: Natural and synthetic compounds, screening and analysis methodologies and future perspectives. Food. Chem. Toxicol., v.51, p.1525, 2013.

DAS, U.N. Acetylcholinesterase and butyrylcholinesterase as possible markers of lowgrade systemic inflammation. Med. Sci. Mon., v.13, p.214-221, 2007.

DESBOROUGH JP. The stress response to trauma and surgery. Br. J. Anaesth., v.85, p.109117,2000

ELLMAN, G.L. COURTNEY, K.D.; ANDRES JR., V.; FEATHERSTONE, R.M. A new and rapid colorimetric determination of acetylcholinesterase activity. Biochem. Pharmacol., v.7, p.88-95, 1961.

FREEMAN, L.J. RAHMANI, E.Y.; RAHMANI, M. et al. Comparison of pain and postoperative stress in dogs undergoing natural orifi ce transluminal endoscopic surgery, laparoscopic, and open oophorectomy. Gastrointest. Endosc., v.72, p.373-380, 2010.
GUVEN, S. MUCI, E.; UNSAL, M.A. et al. The effects of carbon dioxide pneumoperitoneum on ovarian blood flow, oxidative stress markers, and morphology during laparoscopy: a rabbit model. Fertil. Steril., v.93, p.1327-1332, 2010.

JAIN, N.C. Essentials of veterinary hematology. Philadelphia: Lea e Febiger, 1993. 417p.

JENTZSCH, A.M.; BACHMANN, H.; FURST, P.; BIESALSKI, H.K. Improved analisys of malondialdehyde in human body fluids. Free Radic. Bio. Med., v.20, p.251-256, 1996.

KANEKO, J.J.; HARVEY, J.W.; BRUSS, M.L. Clinical biochemistry of domestic animals. 6.ed. Sandiego: Elsevier, 2008. p.799-810.

KIM, P.K.; DEUTSCHMAN, C.S. Inflammatory responses and mediators. Surg. Clin. N. Am., v.80, p.885-894, 2000.

KÜCÜKAKIN, B.; GÖGENUR, I.; REITER, R.J.; ROSENBERG, J. Oxidative Stress in Relation to Surgery: Is There a Role for the Antioxidant Melatonin? J. Surg. Res., v.152, p.338-347, 2009.

LEE, J.Y.; KIM, M.C. Comparison of oxidative stress status in dogs undergoing laparoscopic and open ovariectomy. J. Vet. Med. Sci., v.76, p.273276, 2014.

MACPHAIL, M.C. Surgery of the reproductive and genital systems. In: FOSSUM, T.W. Small animal surgery. 4.ed. St. Louis: Mosby, 2013. p.780-855.

MASSONE, F. Planos anestésicos. In: Anestesiologia veterinária: farmacologia e técnicas. 5.ed. Rio de Janeiro: Guanabara, 2011. p.46-52.

MEYER, D.J. Medicina de laboratório veterinária: interpretação e diagnóstico. São Paulo: Roca, 1995. p.27-29.

MURUGAN, P.; PARI, L. Influence of tetrahydrocurcumin on erytrocytre membrane bound enzymes and antioxidant status in experimental type 2 diabetic rats. $J$. Ethnopharmacol., v.113, p.479-486, 2007.

NELSON, D.L.; KIESOW, L.A. Entalpy of the composition of hydrogen peroxide by catalase at $25^{\circ} \mathrm{C}$. Anal. Biochem., v.49, p.474-479, 1972.

R CORE Team. R: a language and environment for statistical computing. Vienna, Austria: R Foundation for Statistical Computing, 2014. 
REBAR, A.H. Hemogram interpretation. In: Hemogram interpretation for dogs and cats. Missouri: The Gloyd Group, 2004. p.33-38.

RIZZI, T.E.; MEINKOTH, J.H.; CLINKENBEARD, K.D. Normal hematology of the dog. In: WEISS, D.J.; WARDROP, K.J. Schalm's veterinary hematology. 6.ed. Iowa: Blackwell Publishing Company, 2010. p.799-810.

SCHULTZE, A.E. Interpretation of canine leukocyte responses. In: WEISS, D.J.; WARDROP, K.J. Schalm's veterinary hematology. 6.ed. Iowa: Blackwell Publishing Company, 2010. p.321-334.

SOUZA, A.M.B. ROGERS, M.S.; WANG, C.C. et al. Comparison of peritoneal oxidative stress during laparoscopy and laparotomy. J. Am. Assoc. Gyn. Lap., v.10, p.65-74, 2003.

STOCKHAM, S.L.; SCOTT, M.A. Leucocytes. In: . Fundamentals of veterinary clinical pathology. 2.ed. Iowa: Blackwell Publishing Company, 2002. p.53-106.
THOMAS, S.; BALASUBRAMANIAN, K.A. Role of intestine in postsurgical complications: involvement of free radicals. Free Radic. Bio. Med., v.36, p.745-756, 2004.

TRALL, M. A. Hematologia e bioquímica veterinária. São Paulo: Roca, 2007. p.135.

ULLOA, L. The vagus nerve and the nicotinic anti-inflammatory pathway. Nat. Rev. Drug Discov., v.4, p.673-684, 2005.

VAJDOVICH, P. Free radicals and antioxidants in inflammatory processes and ischemiareperfusion injury. Vet. Clin. N. Am. Small, v.38, p.31-123, 2008.

VASCONCELOS, S.M.L. GOULART, M.O.F.; MOURA, J.B.F. et al. Espécies reativas de oxigênio e de nitrogênio, antioxidantes e marcadores de dano oxidativo em sangue humano: principais métodos analíticos para sua determinação. Quim. Nova, v.30, p.1323-1338, 2007.

WOREK, F.; MAST. U.; KIDERLEN, D. et al. Improved determination of acetylcholinesterase activity in human whole blood. Clin. Chim. Acta, v.288, p.73-90, 1999. 